\title{
BMJ Open Evaluation for the safety and effectiveness of the in situ fenestration system in TEVAR for aortic arch pathologies: protocol for a prospective, multicentre and single-arm study
}

Xiaofan Sun, ${ }^{1}$ Yuanqing Kan, ${ }^{1}$ Lihong Huang, ${ }^{1,2}$ Zhihui Dong, ${ }^{1}$ Daqiao Guo, ${ }^{1}$ Yi Si (D), ${ }^{1}$ Weiguo $\mathrm{Fu}^{1}$

To cite: Sun $X$, Kan Y, Huang L, et al. Evaluation for the safety and effectiveness of the in situ fenestration system in TEVAR for aortic arch pathologies: protocol for a prospective, multicentre and single-arm study. BMJ Open 2021;11:e043599. doi:10.1136/ bmjopen-2020-043599

- Prepublication history for this paper is available online. To view these files, please visit the journal online (http://dx.doi. org/10.1136/bmjopen-2020043599).

$\mathrm{XS}$ and YK contributed equally.

Received 14 August 2020 Revised 06 January 2021 Accepted 13 January 2021

Check for updates

(c) Author(s) (or their employer(s)) 2021. Re-use permitted under CC BY-NC. No commercial re-use. See rights and permissions. Published by BMJ.

${ }^{1}$ Department of Vascular Surgery, Zhongshan Hospital Fudan University, Shanghai, China

${ }^{2}$ Department of Biostatistics, Zhongshan Hospital Fudan University, Shanghai, China

Correspondence to

Dr Yi Si;

si.yi@zs-hospital.sh.cn and

Dr Weiguo Fu;

fu.weiguo@zs-hospital.sh.cn

\section{ABSTRACT}

Introduction Thoracic endovascular aortic repair (TEVAR) has gradually become the mainstream therapy for aortic arch pathologies (AAP). Our centre developed the in situ fenestration (ISF) system according to years of clinical experience and technological innovation. This study aims to evaluate the safety and effectiveness of the innovative, self-developed ISF system in TEVAR for AAP.

Methods and analysis The study is a prospective, multicentre and single-arm study. Patients diagnosed with AAPs in five centres on 1 January 2021 will be recruited and general TEVAR with ISF system will be performed. Clinical information and CT angiography images will be collected and recorded. Patients will be followed up for 5 years. Safety and efficacy endpoints are planned to be reported to evaluate this self-developed ISF system.

Ethics and dissemination The study is a registry. We have registered the study on the Chinese Clinical Trial Registry website (http://www.chictr.org.cn/). This study has been approved by the Ethics Committee of Zhongshan Hospital Fudan University (B2020-371) and individual consents will be signed at the time of enrolment. We anticipate that this self-developed ISF system will result in favourable social and economic benefits. Findings will be disseminated in peer-reviewed journals to provide reference for future clinical practice.

Trial registration number ChiCTR1900026696.

\section{INTRODUCTION}

Aortic arch pathologies (AAP), including aortic arch aneurysm and type $\mathrm{B}$ aortic dissection involving aortic arch, are a very dangerous class of aortic diseases for which open surgery is the only effective treatment option in the past. ${ }^{1}$ Since the advent of thoracic endovascular aortic repair (TEVAR), it has gradually been accepted as a great therapy for AAP due to its minimally invasive features with fewer complications and lower mortality rate. ${ }^{2-4}$ Yet, the preservation or reconstruction of the supra-arch arteries poses constant challenges in the endovascular era. ${ }^{5}$ Traditionally,

\section{Strengths and limitations of this study}

- The study is a prospective, multicentre and singlearm study.

- This is the first study focusing on our self-developed in situ fenestration system.

- Comprehensive assessment of safety and effectiveness is carried out in this study.

- The study mostly focuses on Asians resulting in regional limitations.

- There is no comparison group in the study.

hybrid aortic arch repair was done for AAP with supra-arch arteries involved, though with high mortality and non-negligible incidence of neurological complications. ${ }^{6}$ Different techniques have been attempted in the past to reconstruct the supra-arch arteries in such cases, such as branched TEVAR, chimney techniques, in situ laser fenestration techniques, prefenestration techniques and other in situ fenestration (ISF) techniques. ${ }^{7-13}$ The advantages of these techniques are a greater convergence to the normal anatomical morphology and the avoidance of open surgery, which makes total endovascular repair possible. But all these techniques have their own limitations. Custom-made branched endografts are expensive and lack promptness. ${ }^{8}$ Physician-modified fenestrated endografts and prefenestration require a more detailed knowledge of stent-graft and deployment mechanisms. ${ }^{8}$ In situ laser fenestration usually faces technical and devicerelated failures ${ }^{11}$ while needle ISF requires accuracy and stability when puncturing the stent-graft. ${ }^{12}$ Besides, postoperative adverse event rate was still concerning in spite of various attempts. ${ }^{14-16}$ More satisfying and safer approaches were thus urged to arise. 
Based on the existed techniques, our centre developed the ISF system according to years of clinical experience and technological innovation to preserve the supra-arch arteries. We had conducted a single-centre pretest for the device on 1 November 2019. Twelve patients underwent TEVAR with ISF. The rate of instant technical success was $100 \%$, and only one patient suffered a stroke after surgery. The rate of survival without major adverse events (MAE) is $91.7 \%$. The result is satisfied. The device is being audited by China Food and Drug Administration. This study will evaluate the safety and effectiveness of this self-developed ISF system in preparation for its dissemination to maximise patients' benefits with AAPs.

\section{OBJECTIVES}

The objective of the registry is to evaluate the safety and effectiveness of the self-developed ISF system in TEVAR for the AAPs. Primary efficacy endpoint is the rate of instant technical success (successful puncture and branch stent implantation). Primary safety endpoint is the rate of survival without MAE related to procedure or devices in 30 days, including aortic-related death, stroke, respiratory failure, renal failure, paraplegia, intestinal ischaemia and cardiovascular and vascular complications. Secondary safety endpoint is the rate of survival without MAE in the 12-month follow-up and 5-year follow-up.

\section{METHODS \\ Study design}

The study is a prospective, multicentre and single-arm study. Patients admitted with AAPs on 1 January 2021 who met the inclusion criteria will be invited to participate in this study. All enrolled patients will undergo CT angiography (CTA) and clinical evaluation at 30 days, 3 months, 6 months, 12 months and each year after the operation. Clinical information will be acquired through the electronic medical record and the CTA Digital Imaging and Communication in Medicine files from radiology department databases.

This study is designed and initiated by the Department of Vascular Surgery, Zhongshan Hospital Fudan University. We invited four additional participating centres across China with enough TEVAR experience to join this study in order to increase the reliability and generalisability of study results. The participating centres include Taizhou Hospital of Zhejiang Province; Hwa Mei Hospital, University of Chinese Academy of Sciences; Zhejiang Provincial People's Hospital; and The Second Affiliated Hospital of Nanchang University. All their staff involved in the procedure have reached the standard of practice after passing the simulation test.

\section{Participants}

We plan to recruit 65 Chinese patients with high open surgery risk on 1 January 2021 to undergo general TEVAR with self-developed ISF system by surgeons. ${ }^{17}$ Recruitment is non-competitive and will not influence clinical practice. Each of the centres will recruit 12 participants at least.

\section{Criteria}

Inclusion criteria

1. Patients aged 18-80.

2. Patients who are diagnosed with AAPs with involvement of the supra-arch branches.

3. Patients who understand the purpose of the study, willing to participate and sign the informed consent form (ICF) by themselves or their legal representatives.

4. Patients with good compliance who are able to complete follow-up.

\section{Exclusion criteria}

1. Patients allergic to iodinated contrast media.

2. Patients without appropriate arterial access.

3. Patients with a history of myocardial infarction, transient ischaemic attack or cerebral infarction within the past 3 months.

4. Patients with severe concomitant organ failure or malignant tumours.

5. Patients with a history of previous aortic intervention.

6. Patients who are pregnant, lactating or preparing for pregnancy.

7. Patients with connective tissue disease. ${ }^{17}$

8. Patients with a proximal or distal landing zone length less than $25 \mathrm{~mm}$ or a maximum diameter more than $38 \mathrm{~mm}^{.17}$

9. Patients with concomitant aortic valve pathology or high risk for retrograde type A aortic dissection. ${ }^{17}$

10. Patients with a life expectancy of less than 1 year.

11. Patients present with emergent situation, such as aortic rupture.

12. Patients with contralateral carotid/vertebral artery stenosis.

\section{Withdraw criteria}

1. Patients voluntarily request to withdraw.

2. Investigators consider it necessary for subjects to withdraw from a therapeutic standpoint.

3. Patients lost to follow-up.

\section{Endovascular procedure and device design}

General standard TEVAR procedure will be performed in enrolled patients. Self-developed ISF system will then be delivered to target region. After successful fenestration, branch stent will be released to achieve the goal of reconstruction of supra-arch artery.

During the surgery, there are some measures to reduce the risk of neurological complications. First, patients with contralateral carotid/vertebral artery stenosis will be excluded. Cerebral oxygen saturation will be monitoring during the surgery. Sheath bypass or extracorporeal circulation will be performed if necessary. Second, the time of anchoring balloon dilation should be less than 1 min. If puncture fails, the balloon shall be released for $10-15 \mathrm{~min}$. Frequency of puncture must be limited 


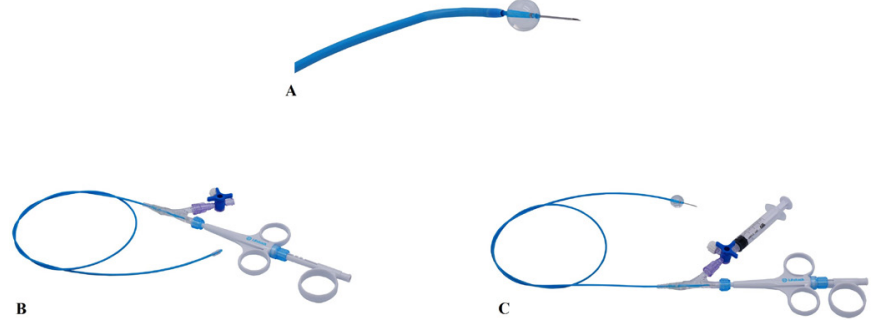

Figure 1 The puncture device of the in situ fenestration system. (A) Details of the puncture needle and the expandable anchoring balloon. (B) Resting status of the puncture device. (C) Working status of the puncture device.

in three times. Last, blood pressure of patients must be increased to ensure blood supply to brain.

When fenestration fails, some bailout procedures will be taken. Device defect and MAE will be reported in writing to ethics committee and department of clinical trial management. As for further treatment, chimney surgery is the first choice. Debranch and bypass surgery or best medical treatment are alternative therapies. If puncture led to vessel perforation, another stent-graft would be placed.

The puncture device of our ISF system (figure 1) includes a puncture needle, an expandable anchoring balloon, a catheter running through the proximal and distal ends of the balloon and a puncture needle that is retractable in the catheter. In the inflated state, the central region of the distal end of the balloon is recessed towards the proximal end to form a recessed region, and the distal end of the catheter is located in the recessed region. This device can improve puncture accuracy and success rate. Besides, the balloon can be delivered along the guide wire to expand the puncture port after withdrawing the puncture needle and push rod. Because the distal end of the anchoring assembly presses against the punctured area, the possibility of the puncture needle puncturing the contralateral covering or the vessel wall is relevantly low.

\section{Study procedure}

The study will be conducted according to the following procedures (figure 2 ):

1. Patients diagnosed with AAPs.

2. Signing the ICF.

3. Subjects screening.

4. Subjects enrolled.

5. TEVAR treatment with ISF system.

6. Follow-up.

\section{Data collection}

We have already designed a standard case report form (CRF) to collect data from electronic medical record. It includes demographics and disease history, surgical information, imaging features, laboratory and other test and medications, and outcomes. Data will be uploaded into electronic CRF (eCRF) by researchers.

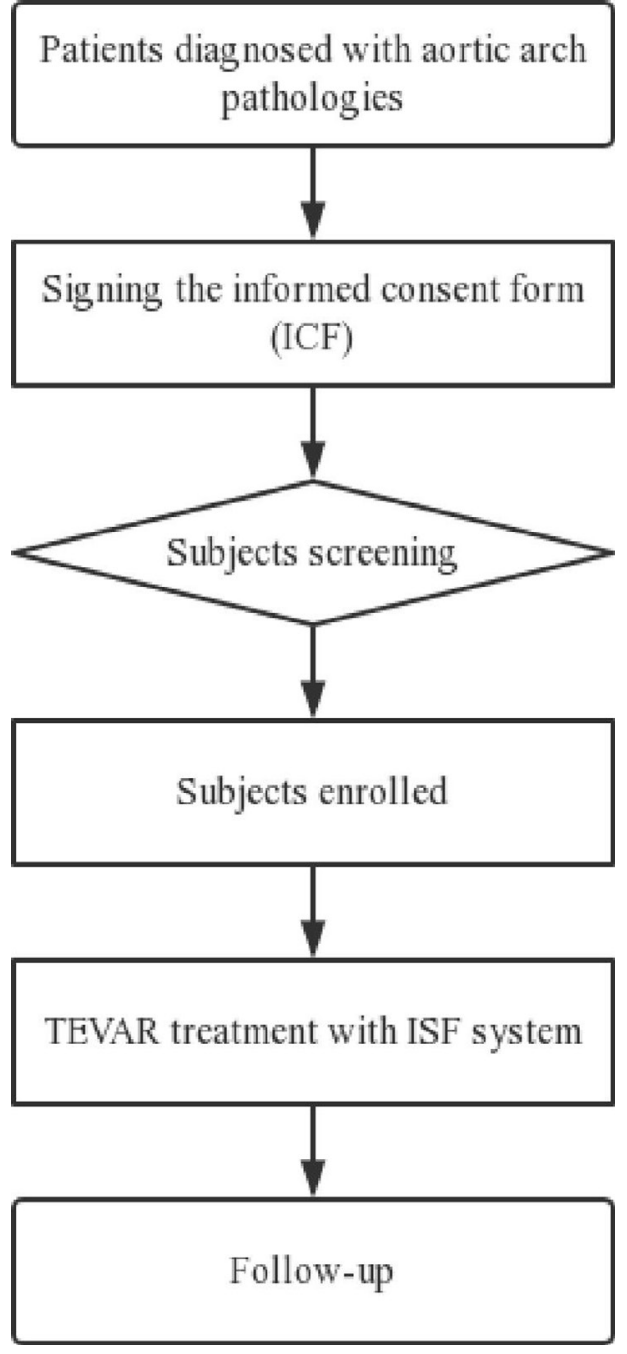

Figure 2 Schematic flow chart of the study. ISF, in situ fenestration; TEVAR, thoracic endovascular aortic repair.

Demographics, comorbidities and perioperative information will be collected during hospitalisation. Imaging features will be analysed through CTA, and CTA will be performed before operation and at 30 days, 3 months, 6 months and each year after operation. All the laboratory and other test will be done before operation. Besides, test of vital signs, blood routine, urine routine, and kidney and liver functions will be done again before discharge.

\section{Data elements}

In order to describe the clinical information and outcomes, our CRF obtains five parts. ${ }^{18}$ Details are as follows:

1. Demographics and comorbidities: age, gender, height, weight, tobacco habits, drink habits, family history, history and comorbidities (including hypertension, diabetes, dyslipidaemia, ischaemic stroke, coronary artery disease, chronic obstructive pulmonary disease, renal insufficiency, atrial fibrillation confirmed by ECG, Marfan syndrome and peripheral vascular disease).

2. Perioperative information: clinical diagnosis (aortic arch aneurysm or aortic dissection), duration of the 
disease (acute, subacute and chronic), impending rupture, pleural effusion, resistant hypertension, resistant pain, ischaemia (spinal cord, organs or lower limb), number, brand and size of stent-grafts, proximal landing zone, endoleak during procedure, conversion to open surgery.

3. Imaging features: classification of dissection, classification of aortic arch, location of primary tear in dissection cases, diameter of proximal landing zone and maximum aneurysm diameter, branch arteries involvement.

4. Laboratory and other test: vital signs (heart rate, breathing, blood pressure and body temperature), ECG, echocardiography, blood routine (red cells, white cells, platelet and haemoglobin), urine routine $(\mathrm{pH}$, white cells, red cells and protein), kidney and liver functions (creatinine, alanine transaminase and aspartate transaminase) and coagulation (activated partial thromboplastin time, international normalised ratio, prothrombin time, fibrinogen and Ddimer).

5. Outcomes: postoperative imaging features and MAE (death, stroke, respiratory failure, renal failure, paraplegia, intestinal ischaemia, cardiovascular and vascular complications) in 30 days, 12 months and 5 years.

\section{Sample size and statistical analysis}

The study selects the rate of technical success as the primary efficacy endpoint. Combined with the current experience of clinical experts, it is estimated that the success rate of ISF surgery is $83 \%$ (objective performance) and technical success rate of experimental ISF system is 97\% (target). ${ }^{19} 20$ According to statistical requirements, we used 0.025 as $\alpha$ and 0.20 as $\beta$ for a single-sided test. Sample size of 40 cases was thus obtained.

Besides, the rate of survival without MAE in 30 days is the primary safety endpoint. The objective performance is set to $88 \%$ and the target of the ISF system is set to $73 \%$. As $\alpha=0.025$ and $\beta=0.2$, sample size equals 59 .

Both of the endpoints need to be achieved. Considering the shedding, the sample size is increased by $10 \%$. At least 65 subjects are required for this study eventually.

After enrolment, all subjects will be divided into three data sets: full analysis set (FAS), per-protocol set (PPS) and safety set (SS). For demographic and baseline information, quantitative data will be used to calculate the number of cases, mean, SD, median, minimum and maximum. Categorical data and ranked data will be used to calculate frequency and composition ratio.

For primary safety endpoint, we use SS analysis to calculate the rate of survival without MAE in 30 days and its 95\% CIs, which is required to be no less than $73 \%$. For efficacy endpoint, PPS and FAS analysis are conducted. The rate of instant technical success must be no less than $83 \%$. For secondary safety endpoint, we will calculate the rate of survival without MAE in 12 months and 5 years and list MAE cases. All the data will be analysed by SAS software (V.9.3 or above).

\section{Data management}

The trial uses EpiData for data management. A data management plan is written by data manager (DM). It will be the guiding document for the entire data management process. All processes of data management should be operated in accordance with the time, content and method defined in the plan. The DM also designs the CRF. It defines study procedure, data form name and data items. Researchers will collect data of subjects in accordance with Good Clinical Practice and data management plan requirements and record the data on eCRF accurately, timely, completely and standardly. After all the data are uploaded into the eCRF, main researchers, statistical analysts and DM will audit data and the database. Then the data will be locked by DM and analysed by statistical analysts. Locked data cannot be edited but can be corrected in statistical analysis programme after confirmation. At the end of the trial, DM prepares a data management report based on the actual implementation of the project.

\section{Patient and public involvement}

Patients or the public will not be involved in the design, or conduct, or reporting, or dissemination plans of our research.

\section{ETHICS AND DISSEMINATION}

This study will be conducted in accordance with the provisions of the Declaration of Helsinki. This study has been approved by the Ethics Committee of Zhongshan Hospital and individual consents will be signed by patients or their assignees at the time of recruitment. Any modifications to the protocol will agreed on by the vascular surgery team from Zhongshan Hospital, and audited and approved by ethics committee.

The data of participants will be stored in locked file cabinets and separate server. All the information of participants will be hidden and replaced by code number. The information will not be disclosed without the written permission of the participants.

All the data will be held by the lead team from Zhongshan Hospital and shared by five centres participating in the trial after negotiation.

We anticipate that this self-developed ISF system would reduce intraoperative blood loss and length of stay, resulting in favourable social and economic benefits. The results of our study would be used for comparisons with other supra-arch techniques and guidance for AAP treatment. Findings will be disseminated in peer-reviewed journals to provide reference for future clinical practice.

Contributors XS, YK, LH, DG, ZD, YS and WF planned the study. XS, YK, DG, ZD, YS and WF designed the study. YK, LH, YS and WF provided ethical support. YK and YS registered the study. XS and YK wrote the study protocol and revised the manuscript. XS and YK developed the CRF.

Funding This work was supported by the National Natural Science Foundation of China (grant 81470581 to YS; grant 81903407 to LH).

Competing interests None declared. 
Patient and public involvement Patients and/or the public were not involved in the design, or conduct, or reporting, or dissemination plans of this research.

Patient consent for publication Obtained.

Provenance and peer review Not commissioned; externally peer reviewed.

Open access This is an open access article distributed in accordance with the Creative Commons Attribution Non Commercial (CC BY-NC 4.0) license, which permits others to distribute, remix, adapt, build upon this work non-commercially, and license their derivative works on different terms, provided the original work is properly cited, appropriate credit is given, any changes made indicated, and the use is non-commercial. See: http://creativecommons.org/licenses/by-nc/4.0/.

ORCID iD

Yi Si http://orcid.org/0000-0001-8320-185X

\section{REFERENCES}

1 Bicknell C, Powell JT. Aortic disease: thoracic endovascular aortic repair. Heart 2015;101:586-91.

2 McWilliams RG, Fearn SJ, Harris PL, et al. Retrograde fenestration of endoluminal grafts from target vessels: feasibility, technique, and potential usage. J Endovasc Ther 2003;10:946-52.

3 McWilliams RG, Murphy M, Hartley D, et al. In situ stent-graft fenestration to preserve the left subclavian artery. $J$ Endovasc Ther 2004;11:170-4.

4 Murphy EH, Dimaio JM, Dean W, et al. Endovascular repair of acute traumatic thoracic aortic transection with laser-assisted in-situ fenestration of a stent-graft covering the left subclavian artery. $J$ Endovasc Ther 2009;16:457-63.

5 Noor N, Sadat U, Hayes PD, et al. Management of the left subclavian artery during endovascular repair of the thoracic aorta. $J$ Endovasc Ther 2008;15:168-76.

6 Andersen ND, Williams JB, Hanna JM, et al. Results with an algorithmic approach to hybrid repair of the aortic arch. J Vasc Surg 2013;57:655-67.

7 van Bakel TM, de Beaufort HW, Trimarchi S, et al. Status of branched endovascular aortic arch repair. Ann Cardiothorac Surg 2018;7:406-13.

8 Anton NS, Bruce AP, eds. Rutherford's vascular surgery and endovascular therapy (p1036-68). 9th ed. Philadelphia: Elsevier, 2019.
9 Qin J, Zhao Z, Wang R, et al. In situ laser fenestration is a feasible method for revascularization of aortic arch during thoracic endovascular aortic repair. J Am Heart Assoc 2017;6:e004542.

$10 \mathrm{Li}$ X-R, Tong Y-H, Li X-Q, et al. Total endovascular repair of an intraoperative stent-graft deployed in the false lumen of Stanford type A aortic dissection: a case report. World J Clin Cases 2020;8:954-62

11 Li C, Xu P, Hua Z, et al. Early and midterm outcomes of in situ laser fenestration during thoracic endovascular aortic repair for acute and subacute aortic arch diseases and analysis of its complications. $J$ Vasc Surg 2020;72:30282-2.

12 Xiang Y, Qiu C, He Y, et al. A single center experience of in situ needle fenestration of supra-aortic branches during thoracic endovascular aortic repair. Ann Vasc Surg 2019;61:107-15.

13 Tse LWH, Lerouge S, Bui BT, et al. Radiofrequency perforation system for in vivo antegrade fenestration of aortic stent-grafts. J Endovasc Ther 2010;17:192-8.

14 Riga CV, Bicknell CD, Basra M, et al. In vitro fenestration of aortic stent-grafts: implications of puncture methods for in situ fenestration durability. J Endovasc Ther 2013;20:536-43.

15 Glorion M, Coscas R, McWilliams RG, et al. A comprehensive review of in situ fenestration of aortic endografts. Eur J Vasc Endovasc Surg 2016;52:787-800.

16 Malina M, Sonesson B. In situ fenestration: a novel option for endovascular aortic arch repair. J Cardiovasc Surg 2015;56:355-62.

17 Czerny M, Schmidli J, Adler S, et al. Editor's Choice - Current Options and Recommendations for the Treatment of Thoracic Aortic Pathologies Involving the Aortic Arch: An Expert Consensus Document of the European Association for Cardio-Thoracic Surgery (EACTS) \& the European Society for Vascular Surgery (ESVS). Eur J Vasc Endovasc Surg 2019;57:165-98.

18 Lombardi JV, Hughes GC, Appoo JJ, et al. Society for vascular surgery (SVS) and society of thoracic surgeons (STS) reporting standards for type B aortic dissections. J Vasc Surg 2020;71:723-47.

19 Li C, Xu P, Hua Z, et al. Early and midterm outcomes of in situ laser fenestration during thoracic endovascular aortic repair for acute and subacute aortic arch diseases and analysis of its complications. $J$ Vasc Surg 2020;72:1524-33.

20 Li HL, Chan YC, Jia HY, et al. Methods and clinical outcomes of in situ fenestration for aortic arch revascularization during thoracic endovascular aortic repair. Vascular 2020;28:333-41. 